\title{
Silicon tubes by a closed molten zone: a characterisation study
}

\author{
R.M. Gamboa*, M.C. Brito, J.M. Serra, J. Maia Alves, \\ A.M. Vallera
}

Universidade de Lisboa, Dep. de FísicalCCMM, P-1749-016 Lisboa, Portugal

\begin{abstract}
We report on the characterisation of silicon tubes recrystallised by closed molten zone, a technique developed as a step to a possible process for thin silicon sheet production. The tube faces are quite flat and have a smooth surface. For the electrical characterisation, samples were cut from the tube faces and simple photovoltaic solar cells were formed. The average diffusion length of minority carriers was found, from spectral response, to be around $100 \mu \mathrm{m}$. Lowresolution LBIC measurements showed lower diffusion lengths (around $40 \mu \mathrm{m}$ ) in the regions close to the tube edges. This behaviour was correlated to measurements of residual thermal stresses using infrared photoelasticity. Measurements of the changes of spectral response with increasing bias light intensity reveal an increase in the effective diffusion length, a known effect that is interpreted in terms of a density of trapping states. (C) 2002 Elsevier Science B.V. All rights reserved.
\end{abstract}

Keywords: Silicon; Ribbons; Characterisation; Diffusion length; Stress

\section{Introduction}

One possible strategy for the cost reduction of photovoltaic energy is the production of thin ribbon silicon cells. Thin cells, besides using less material, should allow the use of silicon of lower quality than that required in thick cells, without major reduction in the efficiency [1]. On the other hand, cutting bulk ingots into thin silicon wafers is an expensive, material wasting process step [2] which is avoided in a ribbon growth process.

\footnotetext{
*Corresponding author.

E-mail address: rgamboa@fc.ul.pt (R.M. Gamboa).
} 
In the recent past, the development of thinner silicon ribbons has been carried out by several groups. For instance, Garcia et al. [3] developed a modified EFG process producing cylindrical tubes with $50 \mathrm{~cm}$ diameter, aiming at $100 \mu \mathrm{m}$ thickness. Wallace et al. [4] refer the development of post-heaters to allow the growth of string ribbon silicon with thickness below $125 \mu \mathrm{m}$.

Our study of silicon tube formation from a closed molten zone is part of an effort to develop a process aiming at the production of thin silicon using molten zones [5]. The main advantages of floating molten zones are that crucible contamination (and cost) is avoided and that, at any one moment, only a small amount of silicon is kept liquid, which in principle leads to an overall lower energy process. The use of a closed shape, e.g. a square, avoids edge instabilities and thus simplifies stabilisation of the molten zone.

In this paper we report on the characterisation of silicon tubes grown using the closed molten zone process. Minority carrier diffusion length measurements by Surface Photo-Voltage (SPV), spectral response and LBIC (Light Beam Induced Current) are presented and discussed. A study of the electrical activity of the surface island defects, evaluated using Electron Beam Induced Current (EBIC) is also presented. Finally, the diffusion length measurements are correlated to residual thermal stress, as measured by infrared photoelasticity.

\section{Silicon tubes by a closed molten zone}

The possibility of silicon tube recrystallisation, from pre-ribbons, using a closed molten zone has been demonstrated [6] and several silicon tubes were obtained. The pre-ribbons used were p-type $(0.5-2 \Omega \mathrm{cm})$ Eurosil and Baysix multicrystalline $350 \mu \mathrm{m}$ thick wafers. An example of an as-grown square silicon tube resulting from closed molten zone crystallisation is shown in Fig. 1.

Silicon tube samples for this study were produced only at moderate growth speeds, between 1 and $3 \mathrm{~mm} / \mathrm{min}$.

\section{Characterisation of the silicon tube samples}

\subsection{Structural characterisation}

The crystallised silicon surface is quite flat, showing a $6 \mu \mathrm{m}$ height wave-like profile with $500 \mu \mathrm{m}$ period, as measured by a mechanical profiler (Dektak). The grains grow in a columnar way from the solid next to the molten zone. In the central region, the grain area is between 10 to $50 \mathrm{~mm}^{2}$, while near the edges $(5-8 \mathrm{~mm})$ the grain area is reduced to below $10 \mathrm{~mm}^{2}$, as can be seen in the left part of the Fig. 2. One possible explanation for the smaller grains near the edges is the local bending of the molten zone which induces thermal stress and crystallographic defects. 


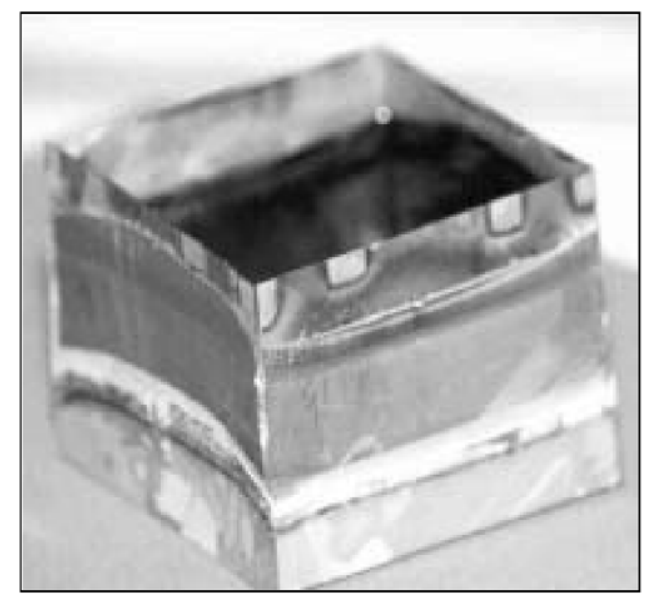

Fig. 1. One of the first as-grown square silicon tubes, with $50 \mathrm{~mm}$ side, resulting from closed molten zone recrystallisation. Oxide marks are visible showing that there was some residual oxygen present in the furnace.
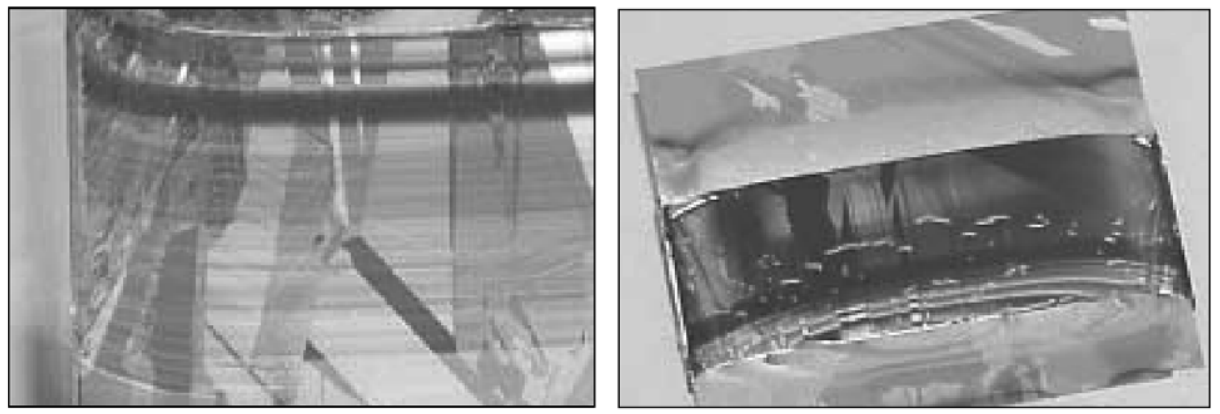

Fig. 2. Photographs showing faces of the recrystallised tubes. The left picture shows the enlargement of the recrystallised region of one half of the tube face. Note that the grain dimension decreases from the central region to the area close to the edge. Right photograph shows the internal face of a square tube where the surface defects organised in bands parallel to the molten zone, the surface island defects, can be seen.

Twining and dislocations are the most common visible crystalline defects of the characterised samples. A profile height of $1-2 \mu \mathrm{m}$ was measured for the twining defects. Some surface defects organised parallel to the molten zone were detected, and denominated surface island defects, as shown in the right part of the Fig. 2. The area associated with this kind of defects is about $1 \mathrm{~mm}^{2}$ with a profile height of about $10-22 \mu \mathrm{m}$. These surface defects can be related to furnace cleaning problems as they correlate with oxide thickness. No interstitial oxide was detected in the samples, as measured by infrared spectroscopy near the $110 \mathrm{~mm}^{-1}$ wave number. Nevertheless 
the presence of some oxide near the silicon surface during the crystallisation could be an explanation for these surface island defects.

\subsection{Electrical characterisation}

The minority carrier diffusion length was measured using Surface Photo Voltage (SPV) on as-grown samples and using spectral response on simple solar cells that were formed on samples cut from the recrystallised tube faces.

\subsubsection{Diffusion length measured by SPV}

In the as-grown samples, cut from the tube faces, measurements of the diffusion lengths were obtained by the SPV technique. The range of measured diffusion lengths was 19-40 $\mu \mathrm{m}$, which seemed far too low in comparison to the values obtained by diode spectral response, as described below. This problem has been recurrently encountered, and so deserved a more detailed study. The conclusion was that the main reason for the relatively low diffusion length values measured with the SPV technique, when compared to those measured in complete cells, is the absence of bias illumination. In fact, the SPV measurements were made without bias light and, as it will be discussed bellow, the diffusion length of these samples increases markedly with bias light intensity.

There can be other reasons for higher diffusion length measurements within a complete cell, of course, such as gettering during junction formation (although such a marked effect would be surprising, given the low temperature and short time involved). However, our study demonstrated that the diffusion length measured in the complete cell with zero bias light is similar to the one obtained for the same asgrown sample by SPV.

\subsubsection{Diffusion length measured by spectral response}

In order to measure the spectral response, simple test cells were made. A procedure with only one thermal step (phosphorous diffusion for junction formation) was used. The procedure for the cell formation can be sequentially presented:

- Sample cutting and cleaning; Solid source phosphorous diffusion at $900^{\circ} \mathrm{C}$ during 30 and 40 min (in a tubular quartz furnace with nitrogen flux), which lead to a sheet resistance around $26 \Omega$ for the $40 \mathrm{~min}$ diffusion and $32 \Omega_{\square}$ for the $30 \mathrm{~min}$ one; Oxide removal and mesa structure formation; Metal contact evaporation (aluminium in the back and $\mathrm{Ti}, \mathrm{Pd}, \mathrm{Ag}$ in the front side) followed by contact annealing.

Spectral response measurements were made and quantum efficiency data was obtained. The diffusion length was obtained from the quantum efficiency data, integrated with the evolution of the absorption coefficient, along the spectrum [7].

The average of the diffusion length values was around $100 \mu \mathrm{m}$, when measured with bias light of $20 \%$ of the sun intensity [5]. It was observed that the diffusion 
length increases with bias light in all analysed samples, behaviour that suggests the existence of defects known as traps. Such defects capture the minority carriers but, once occupied, become less active, until recombination occurs. The bias light used in the cell characterisation leads to a partial or complete saturation of these localised defects thus reducing the effective capture section for the minority carriers and, as result a diffusion length increase is measured.

In order to study the increase of the diffusion length with bias light, several curves of the average quantum efficiency were measured for different bias light intensities, produced using an halogen lamp with a dicroic reflector). The lamp power was kept constant and the distance between the lamp and the cell was adjusted for the different intensities (measured by the short circuit current in a calibrated standard solar cell). A sample result is presented in Fig. 3, showing that the diffusion length increases from $44 \mu \mathrm{m}$ (without bias light) to $150 \mu \mathrm{m}$ (at $100 \%$ of the sun intensity). For values of bias light intensity greater than $70 \%$ of the solar intensity, the diffusion length is constant, and it can therefore be concluded that the localised defects become completely saturated. Qualitatively, the curve shown in Fig. 3 is similar to the one presented by Pauw et al. [8].

\subsubsection{Diffusion length map (2D) by low-resolution LBIC}

In order to obtain diffusion length maps of the test cells, a low-resolution Light Beam Induced Current (LBIC) system was used. For these samples it is especially interesting to measure the diffusion length close to the edge, in order to evaluate the activity of the defects in these region.

In order to compare the diffusion length obtained for the central area of the ribbon with the one measured near the edges, two adjacent cells from the same tube face were characterised by LBIC: cell A (from the central zone) and cell B (adjacent to $\mathrm{A}$ and next to the edge). The LBIC diffusion length maps obtained only with ambient bias light (around $0.1 \%$ of Am1.5 intensity) are shown in Fig. 4.

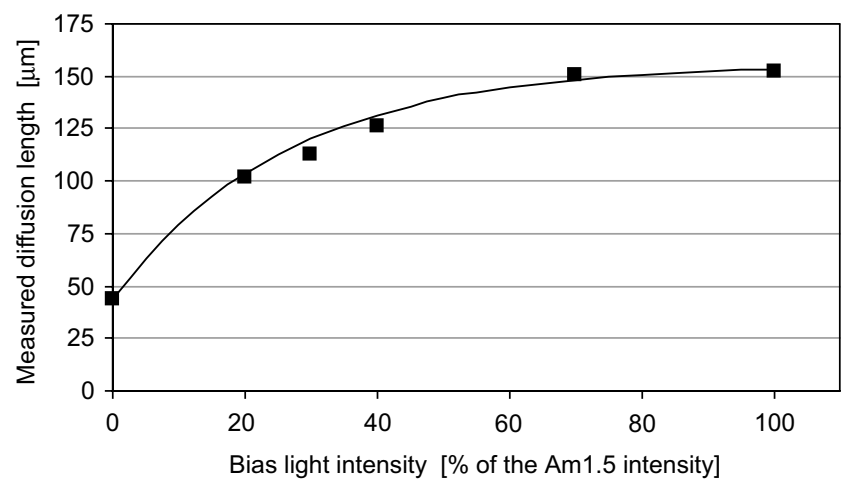

Fig. 3. Diffusion length increase with bias light intensity for one of the test cells made on the samples cut from the recrystallised tube faces material. The line in the graph serves only as a guide to the eye. 
Cell B

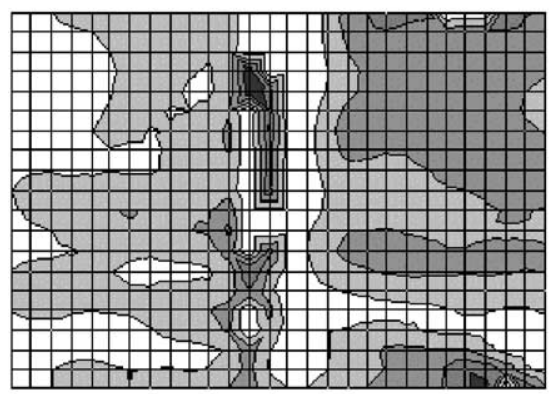

Ln values in micron

$\square 0-40$

$\square 40-80$

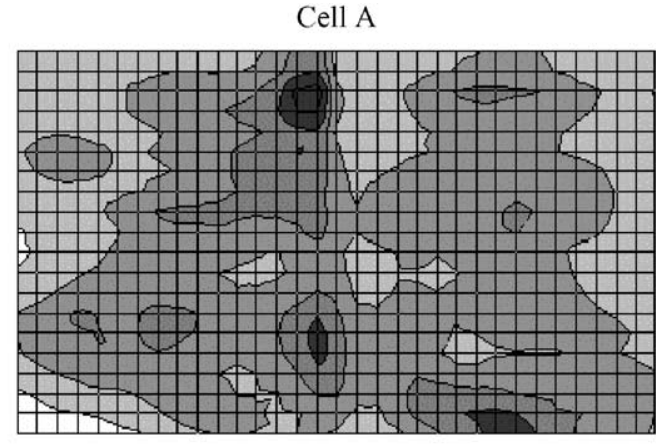

口120-160

$160-200$

Fig. 4. LBIC minority carrier diffusion length ( Ln) maps for cell B (closer to the edge) on the left; and for cell A (central region), on the right. Closer to the edge (left side of the left map), the diffusion length is around $40 \mu \mathrm{m}$ while, for the central region, values near $120 \mu \mathrm{m}$ can be seen. The average diffusion length, obtained without bias light, was $60 \mu \mathrm{m}$ for cell $\mathrm{B}$ and $91 \mu \mathrm{m}$ for cell $\mathrm{A}$. The scanned areas were, respectively, $11 \times 8 \mathrm{~mm}^{2}$ and $13 \times 8 \mathrm{~mm}^{2}$.

The average diffusion length obtained for cell A was $91 \mu \mathrm{m}$. The diffusion length map of cell B reveals the existence of an area, close to the edge, with poorer diffusion length (around $40 \mu \mathrm{m}$ ) than the central region of the tube face. This area, up to approximately $8 \mathrm{~mm}$ from the edge, corresponds to a small grain region (c.f. Section 3.1) and contains a higher density of electrically active defects. The area farther away from the edge has an higher diffusion length resulting in an average diffusion length for the cell B around $60 \mu \mathrm{m}$. The continuity of the areas in relation to the map of the adjacent cell (cell A) can be noted.

\subsubsection{Electrical activity of the surface island defects}

The electrical activity of the surface island defects was evaluated using electron beam induced current (EBIC) images correlated with secondary electron microscope (SEM) images. Results showed that the main grain boundaries in the SEM image are clearly visible in the EBIC image since the collected current is not so high due to an increment of the recombination in the neighbourhood. However, in the regions where the island defects are present there is no measurable reduction of the collected current, leading to the conclusion that these surface island defects are not electrically active.

\subsection{Residual thermal stress}

\subsubsection{Set up}

In order to understand the low diffusion lengths obtained in the edge regions of the ribbons, we initiated the study of the residual thermal stress of the ribbons. The stress measuring system is based on the photoelastic principle that states that certain materials under residual or externally applied stress behave in a birefractive manner. 
The difference between the principal components of the stress tensor (which is the parameter that determines crack initiation [9]) is given by the Maxwell's Stress Optic law

$$
C\left(\sigma_{1}-\sigma_{2}\right)=n_{1}-n_{2}
$$

where $n_{1}$ and $n_{2}$ are the refraction indices along the axes containing the principal stresses and $C$ is the relative stress-optic coefficient, a material property.

When a beam of light travels through the sample, the polarisation of the beam is changed, the angle being proportional to the difference of the principal components of the stress tensor. Using a second polariser, the condition of light extinction can be shown to be

$$
\left(\sigma_{1}-\sigma_{2}\right)=\frac{N \lambda}{t C}
$$

where $t$ is the sample thickness, $\lambda$ is the wavelength and $N=0,1,2, \ldots$ is the fringe order [10].

The developed system, shown in Fig. 5, uses a sinusoidally modulated infrared $(1310 \mathrm{~nm}) 10 \mathrm{~mW}$ laser diode. The laser beam runs through a polariscope consisting of: one polariser and a rotated quarter-wave plate (in order to produce circularly polarised light); the sample holder; a second quarter-wave plate and a second polariser (called analyser). The output is then measured with a detector activated by a lock-in system. A six steps phase shifting method is used to determine the fringe order [11].

\subsubsection{Results}

The thermal stresses of samples of the same tube used to produce the solar cells discussed in Section 3.2 were measured. Preliminary results for line scans throughout a tube face are shown in Fig. 6. As one approach the edges of the tube face, there is a clear increase of the residual stress. By comparing these data with the LBIC results,

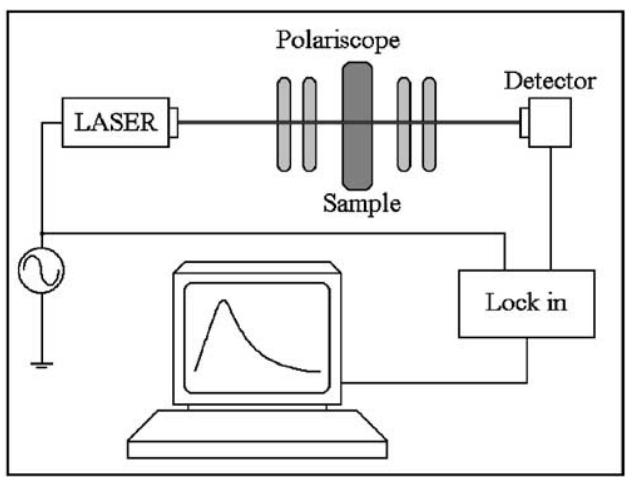

Fig. 5. Diagram of set up for residual stress measurement. 


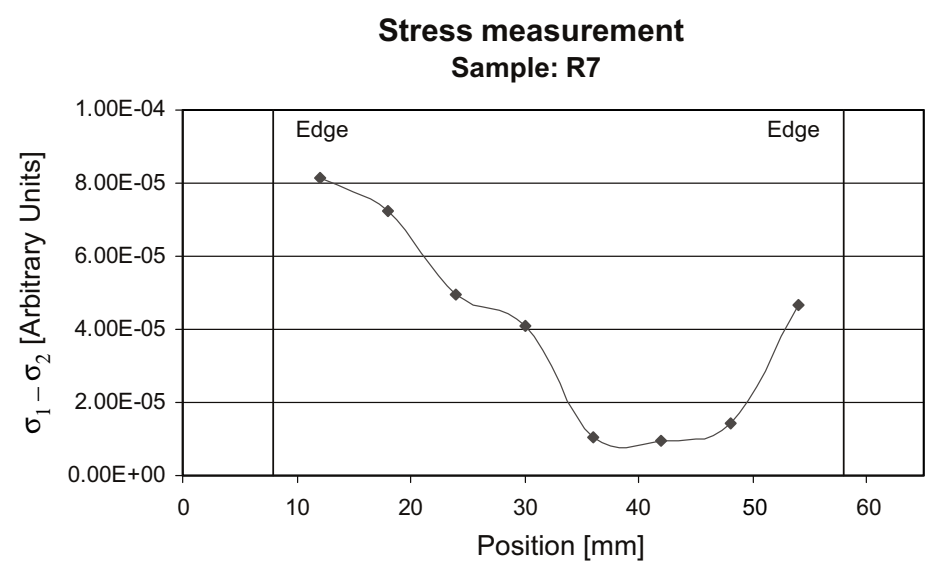

Fig. 6. Preliminary measurements of residual thermal stress of silicon closed molten zone ribbons by infrared photoelasticity. The line scan indicates that the stress increases near the tube face edges, on this sample more at the left side then at the right.

one can conclude that the low diffusion lengths are, in all likelihood, the result of the thermal stresses.

\section{Conclusions}

Silicon ribbons obtained from recrystallisation of square silicon tube using a closed molten zone were characterised. The central zone of recrystallised ribbons present grains of the order of $10-50 \mathrm{~mm}^{2}$, while in the area located about $8 \mathrm{~mm}$ next to the edge the grain size is below $10 \mathrm{~mm}^{2}$. The recrystallised silicon ribbons also present some surface "island" defects and crystallographic defects such as twining and dislocations.

Simple test cells were made from samples cut from the tube faces, with the junction formation as the only thermal step (no passivation, gettering or texturing were performed). The characterisation of the test cells revealed average diffusion length around $100 \mu \mathrm{m}$ measured in the samples from the central zone of the tube faces. Diffusion length maps were obtained using a low resolution LBIC system, revealing that closer to the edges $(8 \mathrm{~mm})$ the diffusion length decreases to $40 \mu \mathrm{m}$. Measurements of the residual thermal stress using infrared photoelasticity showed that the low diffusion length close to the edges is correlated to thermal stress.

An EBIC system was used to evaluate the electrical activity of the island defects. Comparing the SEM image with the one obtained by EBIC it was possible conclude that the surface island defects are not electrically active.

An increment of the diffusion length with bias light intensity was seen in all analysed samples. Values ranging from $44 \mu \mathrm{m}$ (zero bias light) to $150 \mu \mathrm{m}$ ( bias light 
of $70 \%$ of the Am 1.5 intensity) were demonstrated. This effect is associated to a high density of traps that become saturated with the bias light.

\section{Acknowledgements}

This work was partially supported by the European Commission, within the JOULE program, under contracts JOR3-CT 95-0030 and JOR3-CT 98-0287 (DG 12-WSME) and by FCT (POCTI/1999/CTM/34618). One of the authors was supported by PRAXIS XXI grants, which is gratefully acknowledged.

\section{References}

[1] C. Sah, K. Yamakawa, R. Lutwack, IEEE Trans. Electron Dev. 29 (1982) 29.

[2] T. Bruton, et al., Proceedings of the 14th EC PVSEC, Barcelona, 1997, pp. 11-16.

[3] D. Garcia, M. Ouellette, B. Mackintosh, J.P. Kalejs, J. Cryst. Growth 225 (2000) 566.

[4] R. Wallace, R. Janoch, J. Hanoka, Proceedings of the 2nd WC PVSEC, EC, V2, Viena, 1998, pp. $1818-1821$.

[5] R. Gamboa, J.C. Henriques, J.M. Serra, J. Maia Alves, A.M. Vallera, Proceedings of the 16th EC PVSEC, Glasgow, 2000.

[6] R. Gamboa, PhD Thesis, University of Lisbon, 2000.

[7] R.O. Bell, G.H. Freedman, Proceedings of the 13rd IEEE PVSC, 1978, p. 89.

[8] P. de Pauw, R. Mertens, R. Van Overstraten, Materials Processing Theory and Practices, NorthHolland, Amsterdam, Vol. 6, 1987, pp. 5-95.

[9] C. Buckberry, D. Towers, Measur. Sci. Technol. 6 (1995) 1227-1235.

[10] R. Budynas, Advanced strength and applied stress analysis, McGraw Hill, New York, 1977.

[11] M.C. Brito et al., Proceedings of the 17th EC PVSEC, Munich, 2001. 